\title{
Deformability of poly(amidoamine) dendrimers
}

\author{
A. Mecke ${ }^{1,2}$, I. Lee ${ }^{3}$, J.R. Baker jr. ${ }^{2}$, M.M. Banaszak Holl ${ }^{4,2, a}$, and B.G. Orr ${ }^{1,2, b}$ \\ 1 Department of Physics, Harrison M. Randall Laboratory, The University of Michigan, 500 E. University Ave., Ann Arbor, \\ MI 48109-1120, USA \\ 2 Center for Biologic Nanotechnology, The University of Michigan Medical School, 1150 W. Medical Center Dr., Ann Arbor, \\ MI 48109-0648, USA \\ 3 Michigan Center for Biological Information, The University of Michigan Medical School, 3600 Green Court, Suite 700, Ann \\ Arbor, MI 48105, USA \\ 4 Department of Chemistry, The University of Michigan, 930 N. University Ave., Ann Arbor, MI 48109-1055, USA
}

Received 9 June 2003 and Received in final form 25 November 2003 /

Published online: 2 June 2004 - (c) EDP Sciences / Società Italiana di Fisica / Springer-Verlag 2004

\begin{abstract}
Experimental data indicates that poly(amidoamine) (PAMAM) dendrimers flatten when in contact with a substrate, i.e. they are no longer spherical, but resemble flat disks. In order to better understand the deformation behavior of these branched polymers, a series of atomistic molecular dynamics simulations is performed. The resulting flattened dendrimer conformations are compared to atomic force microscopy (AFM) images of individual dendrimers at air/mica and water/mica interfaces. The ability of the polymers to deform is investigated as a function of dendrimer generation (2-5) and the required energies are calculated. Our modeling results show good agreement with the experimental AFM images, namely that dendrimers are highly flexible and capable of forming multiple interaction sites between most of their branch ends and the substrate. The deformation energy scales with dendrimer generation and does not indicate an increase in stiffness between generations 2 and 5 due to steric effects.
\end{abstract}

PACS. 81.07.Nb Molecular nanostructures - 82.20.Wt Computational modeling; simulation - 68.37.Ps Atomic force microscopy (AFM)

\section{Introduction}

Since their introduction about twenty years ago dendrimers have attracted a great deal of scientific attention (for a review see Bosman et al. and references therein [1]). Their well-defined molecular structure, including a large number of functional sites on their branch ends, makes them promising candidates for medical and biological applications. Poly(amidoamine) (PAMAM) dendrimers (Fig. 1), for example, have been enhanced by the attachment of several types of biologic materials. The resulting nanodevices have applications in a number of different areas of biology and medicine such as gene and drug delivery [2-8].

In order to transport a biologic agent into the cell of an organism, the dendrimer has to interact with the cell wall. The exact shape and structure of the macromolecules near membranes may impact their biologic function. For example, distortions of the dendrimer surface can increase the area of contact to a substrate and facilitate the interaction of multiple functional sites. Also, if the dendrimer deforms

\footnotetext{
a e-mail: mbanasza@umich.edu

b e-mail: orr@umich.edu
}

and loses its interior compartments, the ability to transport physically intercalated smaller molecules may be lost. For the design of nanodevices with optimal uptake properties it is therefore essential to understand the structure and behavior of dendrimers in contact with surfaces.

Atomistically flat substrates such as mica have proven to be useful models for this kind of interaction. Like biologic membranes, mica possesses a negative surface charge density. While the actual cell surface is a complex system of many different components, some comparable in size to dendrimers, mica is more readily accessible to high-resolution scanning probe techniques. It can therefore serve as a starting point to study changes in dendrimer structure at surfaces.

Previous experiments have shown that dendrimers are extensively deformed when in contact with a surface. The adsorbed polymers are no longer spherically symmetric but instead resemble flat disks. This has been demonstrated most impressively in atomic force microscopy (AFM) experiments [9-19]. In a theoretical study, Mansfield described lattice Monte Carlo simulations of nodeand-link model dendrimers under the influence of an 


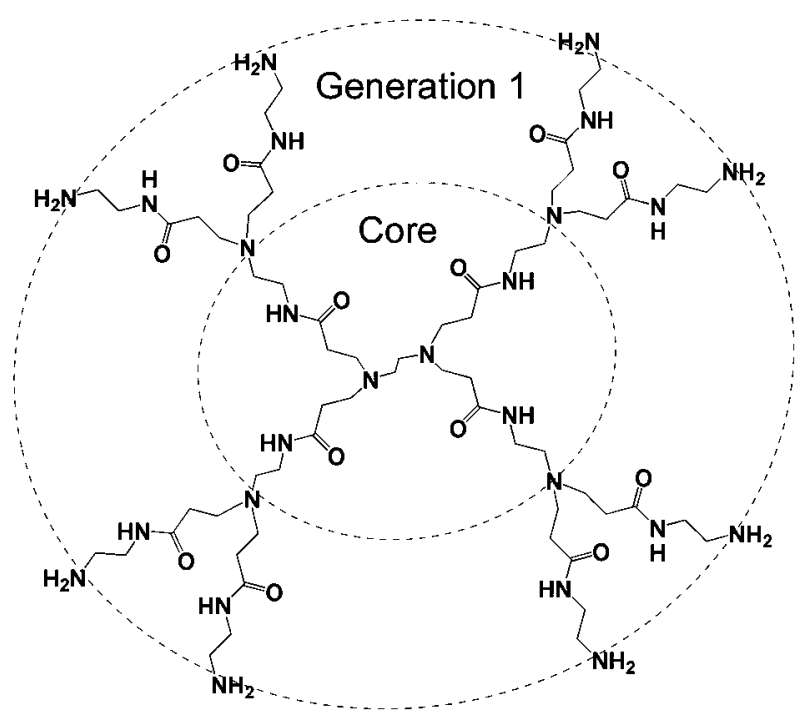

Fig. 1. Generation 1 poly (amidoamine) (PAMAM) dendrimer. Capping groups (in this case $\mathrm{NH}_{2}$ ) can be modified, e.g. to $\mathrm{NHC}(\mathrm{O}) \mathrm{CH}_{3}$ (acetamide).

attractive interaction to a plane and demonstrated flattening of the structures [20].

There are several questions these observations raise that the current paper is trying to address:

1) Does the net charge of branch end groups or the presence of solvent fundamentally influence the deformation behavior of PAMAM dendrimers?

2) Is the experimentally determined shape consistent with an intact dendrimer, i.e. is the backbone of the dendrimer capable of deforming to the experimentally observed degree? Or could the measured topography be the result of an imaging artifact?

3) What does the branching structure of the compressed dendrimer look like? In particular, how does it depend on generation? Where are the branch ends located?

4) What is the energy required to compress the dendrimer to the degree observed in experiment, and how does it depend on dendrimer generation?

The first question is addressed here by performing AFM experiments on PAMAM dendrimers with amineand acetamide-terminated branch ends, respectively, both in air as well as in aqueous solution. The remaining three points are investigated using atomistic molecular dynamics calculations of PAMAM dendrimer models in their spherical as well as compressed state.

The total number of atoms in a dendrimer grows exponentially with its generation, so that simulation models quickly become very large. It is obvious that dendrimer simulations involving the complete crystal structure of muscovite mica and possibly even explicit solvent molecules are computationally demanding. In order to speed up the calculations while still capturing the essential properties of the hyper-branched structure, the deformation behavior of dendrimers is investigated using the following approximations and assumptions: i) The presence of solvent molecules does not prevent the dendrimer from flattening. Also, in most of the abovementioned experiments, the measurements have been performed on dried dendrimers after removing the solvent. Therefore no explicit solvent molecules are used in the simulations presented here. Instead a certain shielding effect of the solvent is introduced by using a dielectric constant (see also Ref. [21]).

ii) The properties of the compressed dendrimer do not depend critically on the type of force causing the deformation. It is thus justified to replace the effect of the substrate by an artificial attractive potential between the dendrimer and a planar surface.

This approach makes it possible to reduce computation time while still retaining all atomistic details of the dendrimer. Comparison of experimental and computational data can then provide a measure for the validity of the above approximations (see discussion section).

Note that the chosen simulation model is not intended to describe the true interaction potential between mica and a polymer. The substrate is used as a tool to deform atomistic models of PAMAM dendrimers to the same degree as observed in experimental studies, and to create a database of dendrimer shapes suitable for conformational analysis using empirical force field parameters. The comparison of the conformational energies of different shapes provides a measure for the stress in a deformed molecule.

The types of dendrimers investigated here have been chosen for their potential as targeted anti-cancer therapeutics [8]. As in vitro investigations of the ability to target dendrimers to specific receptors on cells have shown, the charge of a drug carrier influences its biologic activity. In particular, one can avoid nonspecific uptake by modifying the branch termini of dendrimers. Since primary amines $\left(-\mathrm{NH}_{2}\right.$, shown in Fig. 1$)$ are expected to be protonated at $p \mathrm{H}<7$ they carry a net positive charge $[22,23]$. Thus, in order to take those differences into account, both charged amine capped dendrimers as well as dendrimers with charge neutral acetamide termini $\left(-\mathrm{NHC}(\mathrm{O}) \mathrm{CH}_{3}\right)$ are examined.

Details of the experimental and computational approach can be found in Section 2 of this paper. Section 3 describes the results of the AFM analysis (Sect. 3.1) as well as molecular dynamics simulations (Sect. 3.2). In Section 4 the findings are compared to previous results reported in the literature, and interpreted using geometrical considerations. Finally, Section 5 summarizes the main conclusions.

\section{Experimental and numerical methods}

Poly(amidoamine) (PAMAM) dendrimers of generation 5 and 7 (G5, G7) were provided by the Center for Biologic Nanotechnology, The University of Michigan. Because of their greater size (making them more amenable to AFM imaging) G7 dendrimers were chosen to experimentally study the influence of different functional groups (amine and acetamide) and solvent on their adsorption properties. 

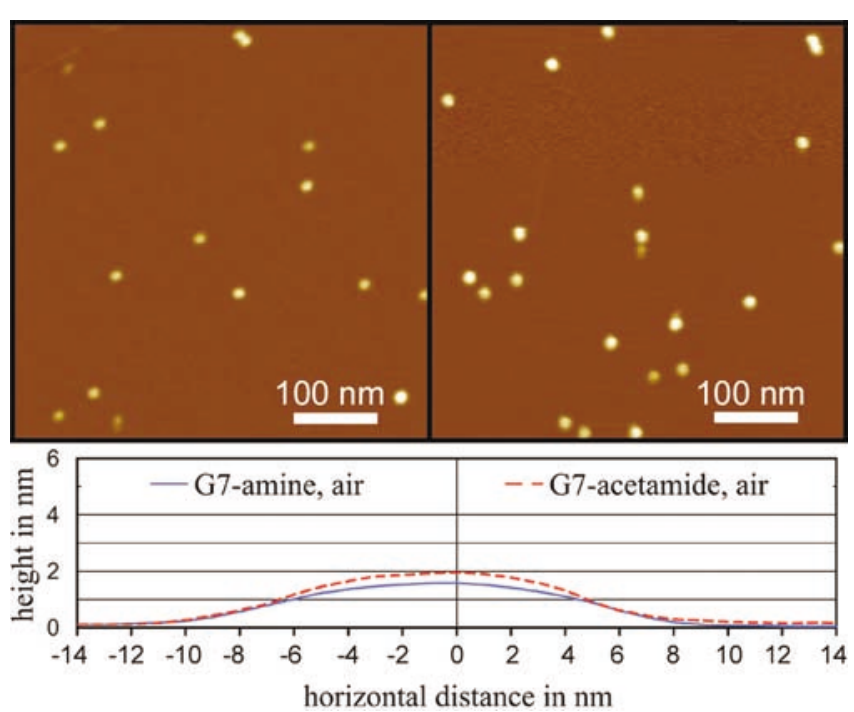

Fig. 2. AFM images of amine-terminated (left) and acetamideterminated (right) G7 PAMAMs on mica in air; below: line scans through the center of two individual dendrimers.

G5 was used to compare shapes of flattened dendrimers observed in experiment vs. simulation.

AFM measurements were performed in tapping mode on a Nanoscope IIIa Multimode scanning probe microscope from Digital Instruments (Veeco Metrology Group, Santa Barbara, CA). The procedure to obtain images of dry dendrimers has previously been described $[17,18]$. Aqueous imaging was carried out using a liquid cell (Digital Instruments) and a silicon nitride cantilever (DI model NPS, spring constant $0.32 \mathrm{~N} / \mathrm{m}$, length $100 \mu \mathrm{m}$ ) at a drive frequency of $6-8 \mathrm{kHz}$. A piece of freshly cleaved mica was first placed on the sample holder and an image of the substrate was taken. Approximately $30 \mu \mathrm{l}$ of a $78 \mathrm{nM}$ aqueous dendrimer solution was then injected into one of the liquid canals of the cantilever holder using a syringe needle. Imaging recommenced in order to observe the adsorption of the dendrimers to the substrate. Note that this injection method was chosen, rather than re-hydrating adsorbed dried dendrimers, to allow for a true competition between hydration and surface adhesion forces during the diffusion and adsorption process.

Computer simulations were performed on an Octane Silicon Graphics workstation as well as on the terascale Compaq Alphaserver Cluster at the Pittsburgh Supercomputing Center. Atomistic models of PAMAM dendrimers were constructed using the Insight II software package by Accelrys Inc., San Diego, CA. Starting with an ethylenediamine core, the dendrimer models were built generation by generation by attaching two monomers to every branch point. The resulting structures were then energy-minimized and relaxed through simulated annealing followed by a molecular dynamics simulation for up to $400 \mathrm{ps}$ until the potential energy flattened out. These initial stages of the simulation were described by Lee et al. [21] and are not included in the data presented here.

The deformation of the dendrimers was achieved by placing the molecules in the confining potential of a two-
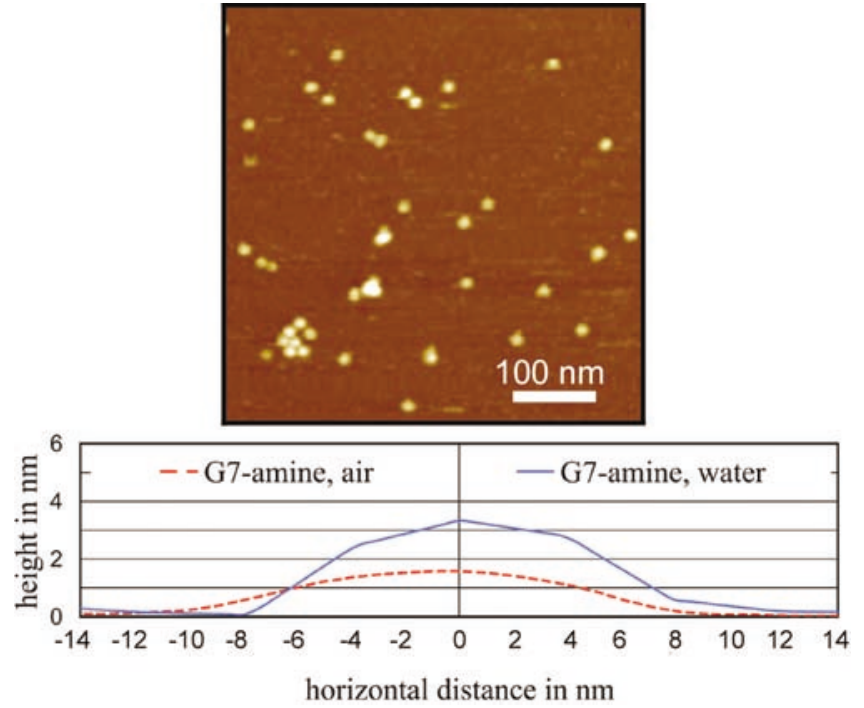

Fig. 3. AFM image of (amine-terminated) G7 PAMAMs on mica in water; below: comparison of line scans in air and water.

dimensional hexagonal array consisting of 1920 atoms, each carrying a negative charge $q$. (Varying the lattice mesh and polarizability does not effect the results.) This attractive plane covered an area of $20 \times 20 \mathrm{~nm}$ (unless otherwise noted), and the positions of the atoms in the plane were held fixed during the simulation. By tuning the parameter $q$ it was possible to increase or decrease the degree of compression of the dendrimer. (As was pointed out in the introduction, $q$ should not be interpreted as an actual surface charge.) The chosen value of the surface charge corresponds to the smallest one that flattened all arms of the dendrimer.

The potential energy expression was set up according to the consistent valence force field (CVFF) [24] including cross terms and a bond stretch potential described by an harmonic term instead of the Morse function. (For the confining potential the fixed atoms in the plane were assigned the same force field parameters as a chlorine ion of equal charge $q$.) Note that the expression for the total potential energy, $E_{\text {pot }}$, of the dendrimer and attractive plane combined includes a dendrimer term, $E_{\text {dendrimer }}$, (i.e. bonded and nonbonded interaction within the dendrimer) as well as the contribution of the confining potential, $E_{\text {confine: }}$ :

$$
E_{\text {pot }}=E_{\text {dendrimer }}+E_{\text {confine }} .
$$

The sole purpose of the second term is to cause the dendrimer to deform, and it has to be excluded when evaluating changes in conformational energy of the dendrimer. All atoms were included when computing the energy expression, i.e. there was no cutoff distance. Although no explicit solvent molecules or counter ions were present in the model, a distance-dependent dielectric constant was introduced in the Coulomb term of the force field expression to account for the shielding of charges by the solvent. The validity of this approach was investigated in reference [21], and is expected to give accurate results for the case of charged dendrimers and less so for the uncharged case. 

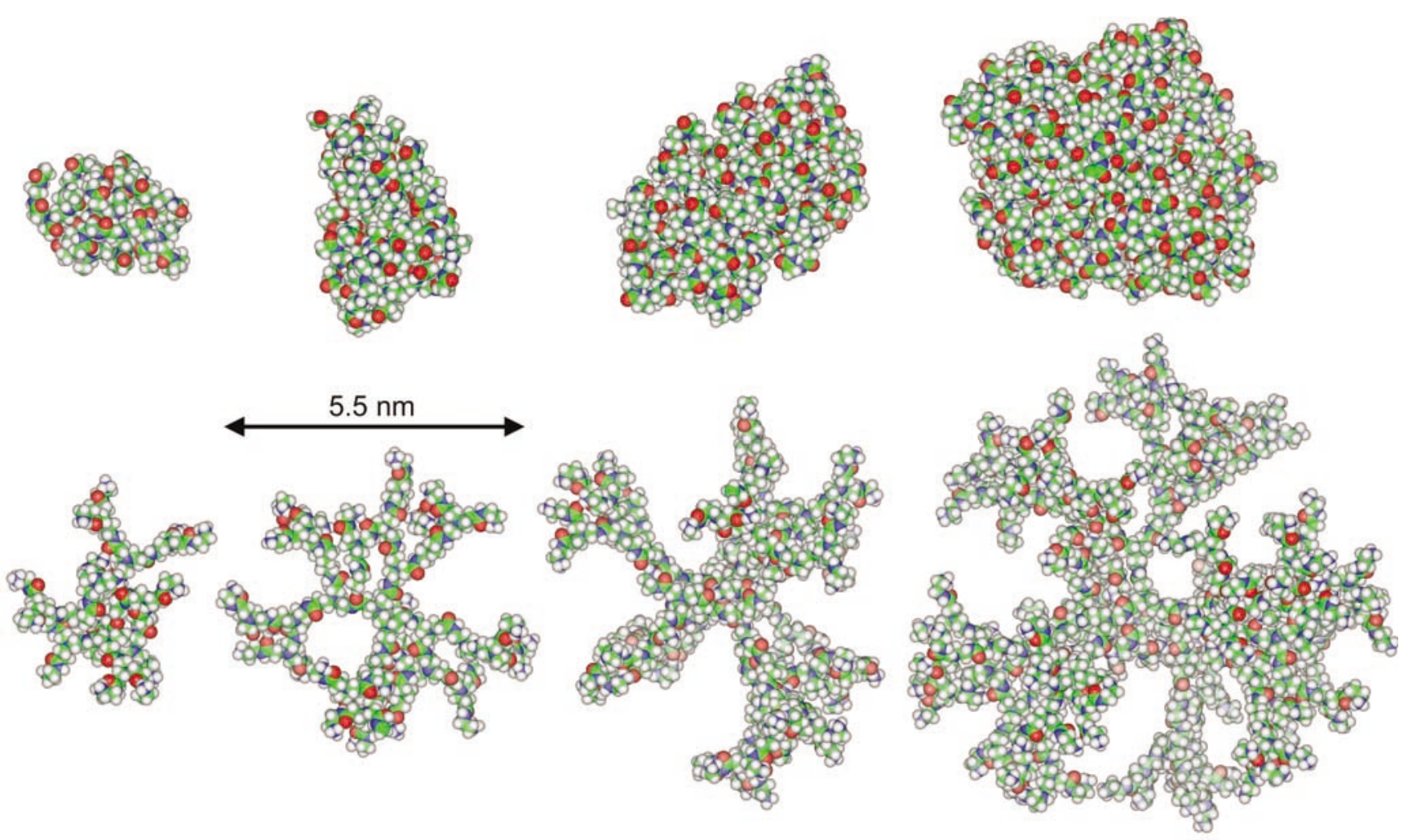

Fig. 4. Top row: Simulation images of free acetamide capped (charge neutral) PAMAMs, generations 2-5. Bottom row: Simulation images of free amine capped (positively charged) PAMAMs, generations 2-5.

Table 1. Theoretical molecular weight (for ideal dendrimer growth) and average size determined from AFM images for G7 PAMAMs.

\begin{tabular}{lccc}
\hline $\begin{array}{l}\text { Capping } \\
\text { group }\end{array}$ & $\begin{array}{c}\text { Molecular } \\
\text { weight }\end{array}$ & $\begin{array}{c}\text { Height } \\
\text { in nm }\end{array}$ & $\begin{array}{c}\text { Volume } \\
\text { in } \mathrm{nm}^{3}\end{array}$ \\
\hline amine (air) & 116491 & $1.4 \pm 0.2$ & $171 \pm 15$ \\
\hline acetamide (air) & 138014 & $1.8 \pm 0.1$ & $244 \pm 19$ \\
\hline amine (water) & 116491 & $2.9 \pm 0.3$ & $419 \pm 42$ \\
\hline
\end{tabular}

Each dendrimer was first simulated in isolation and then under the influence of the confining potential. For the latter case, the initially spherical dendrimer was placed a few $\mathrm{nm}$ above the center of the lattice before starting the simulation. The simulation conditions for dendrimers with or without surface were similar. After a short annealing period, during which the system was heated to $1000 \mathrm{~K}$, the model was then simulated at room temperature for up to $280 \mathrm{ps}$, with a step size of $1 \mathrm{fs}$. The potential energy of the model was monitored throughout the simulation in order to determine the time necessary to reach a stable state. Data collected before this point was excluded from calculating molecular properties such as the radius of gyration, conformational energy, etc.

In addition to that, two different methods were used to test how well the systems were equilibrated at this point. Two of the models, G3- and G5-amine PAMAMs on a surface with $q=-0.5$, were simulated for extended simula- tion times using the simulation package CHARMM [25,26] on 32 parallel processors. This made it possible to observe the evolution of the flattened dendrimer for up to $5 \mathrm{~ns}$ and 2 ns, respectively. The average potential energy after the first 100 ps interval differed by only $2 \%$ from the average potential energy during the last $100 \mathrm{ps}$ interval. Thermal energies were sufficient to allow the branches of the dendrimer to explore a wide range of flattened configurations. This shows that the atomistic details of the artificial deformation potential do not bias the simulations. As a second test, 10 cycles of simulated annealing followed by 100 ps of equilibration were performed, each starting out with a different initial conformation. This method resulted in a greater variety of dendrimer conformations that were then compared to each other. The molecular shapes as well as the average energies remained the same within $0.5 \%$ for all starting conformations tested.

\section{Results}

\subsection{Experimental}

Both amine and acetamide capped dendrimers of generation 5 and 7 assume an oblate shape when adsorbed on a mica surface. Figure 2 shows AFM height images and the corresponding line scans of G7 PAMAMs. (For comparison with computer models, a similar line scan of a G5 PAMAM is used in Fig. 8, see Sect. 3.2). Using the 


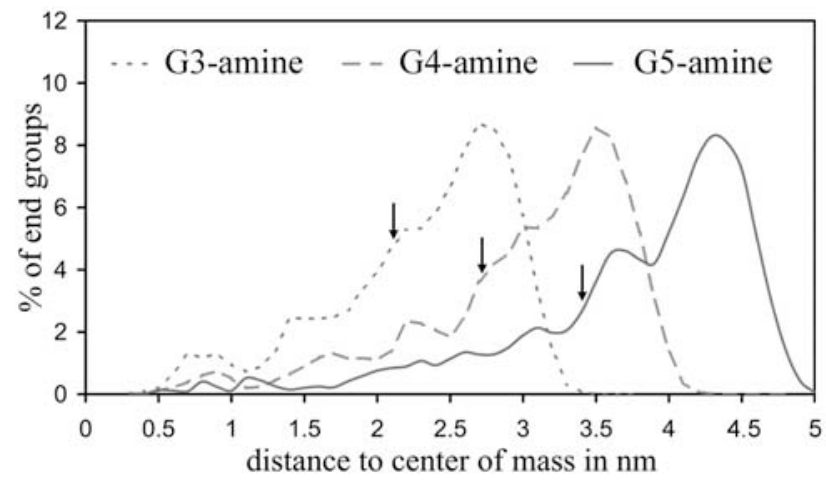

Fig. 5. Distribution of end groups as a function of the distance to the center of mass of the molecule for amine-terminated PAMAMs; arrows indicate radii of gyration.

bearing analysis of the Nanoscope III software it is possible to determine the height and volume of the observed features [17]. This analysis shows a narrow distribution of particle sizes with a standard deviation between $8 \%$ and $10 \%$. Average values for the dendrimers analyzed here are summarized in Table 1. The ideal molecular weight of G7acetamide is higher than for G7-amine, and therefore they are expected to have a greater average volume. The average measured height of acetamide dendrimers is slightly larger than for amine dendrimers, but in both cases it is significantly smaller than the diameter of the spherical dendrimer [27]. In summary, the adsorption behavior on mica surfaces is not influenced significantly by replacing amine branch ends with acetamide ones.

Since dendrimers in biologic systems act in an aqueous environment, the same measurements were performed on G7-amine PAMAMs on mica in water, see Figure 3. As before, a narrow distribution of particle sizes is observed. The experimentally determined values for height and volume increase in water, but one still observes significant flattening of the adsorbed dendrimers even in the presence of solvent.

\subsection{Computational}

In order to obtain a better understanding of this experimentally observed behavior of dendrimers, molecular dynamics simulations of PAMAM dendrimers of generations G2 through G5 are presented. As discussed earlier, models with protonated amine branch ends (denoted as G\#amine) as well as models with the amines replaced by charge neutral acetamide groups (denoted as G\#-acet), see Figure 4, are investigated. From this figure it is immediately evident that electrostatic interactions between charged amine groups prevent most branch ends from folding back into the interior of this molecule resulting in a more open structure with the end groups exposed to the environment. On the other hand, in the acetamide capped PAMAMs there is no such strong repulsive force that prevents the branch ends from populating the entire volume of the molecule.

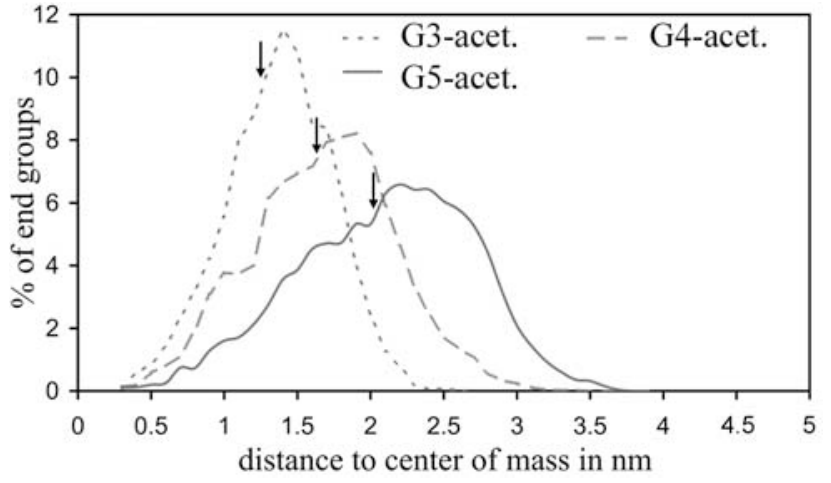

Fig. 6. Distribution of end groups as a function of the distance to the center of mass of the molecule for acetamide-terminated PAMAMs; arrows indicate radii of gyration.
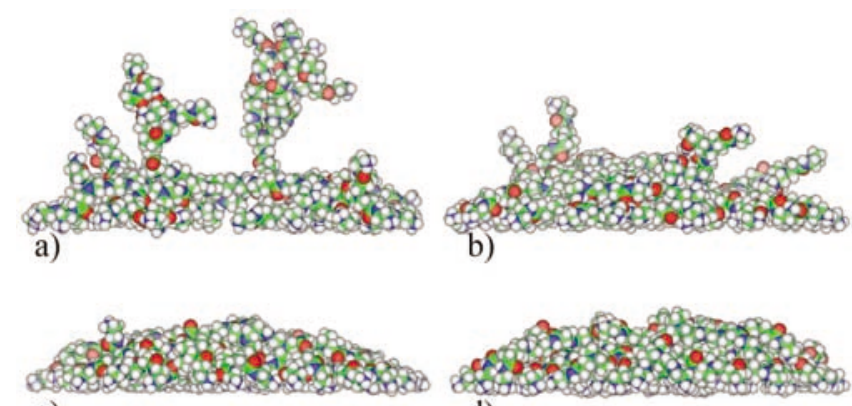

c)

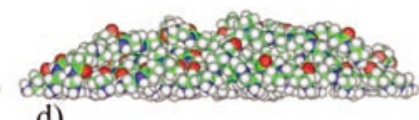
d)

Fig. 7. Shape of G4-amine PAMAM as a function of parameter q. a) $q=-0.05$, b) $q=-0.1$, c) $q=-0.25$, d) $q=-0.5$.

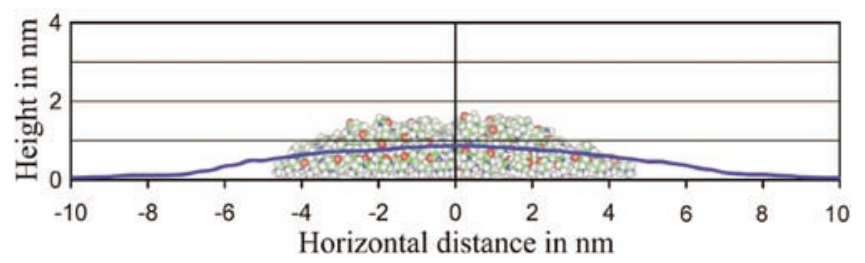

Fig. 8. Line scan taken from AFM image of G5-amine PAMAM on mica in air superimposed on simulation image of flattened G5-amine $(q=-0.5)$.

As a result, the density of end groups in the core region as well as the overall density is higher in the acetamide PAMAMs. This can most easily be demonstrated by plotting the distribution of end groups as a function of the distance to the core averaged over a number of molecular conformations, see Figures 5 and 6. For amine-terminated PAMAMs (Fig. 5) most of the functional groups lie close to the surface of the molecule. In contrast, the uncharged acetamide dendrimers (Fig. 6) are generally smaller and have a higher density near the core due to branches reaching back into the interior of the molecule. This observation as well as the computed radius of gyration for generation 5 agrees well with recent GPC data by Majoros et al. [28].

Turning to the simulations of deformed dendrimers, Figure 7 shows the molecular configuration for different strengths of the confining potential. Figure $7 \mathrm{~d}$ represents 
a)

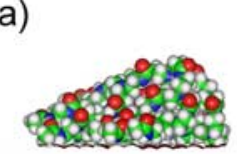

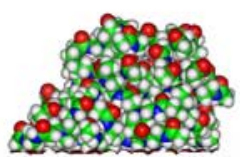
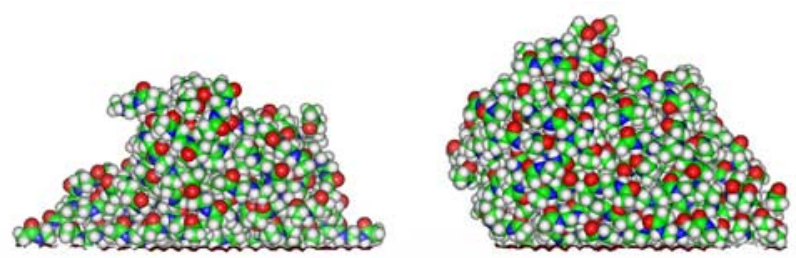

$9.6 \mathrm{~nm}$

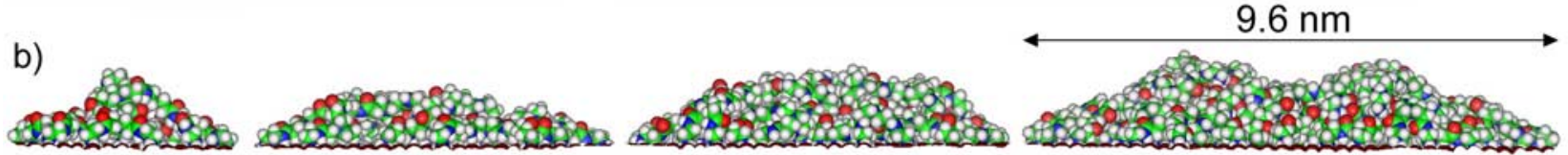

Fig. 9. Simulation images of flattened $(q=-0.5)$ acetamide (a) and amine (b) PAMAMs, generations $2-5$.

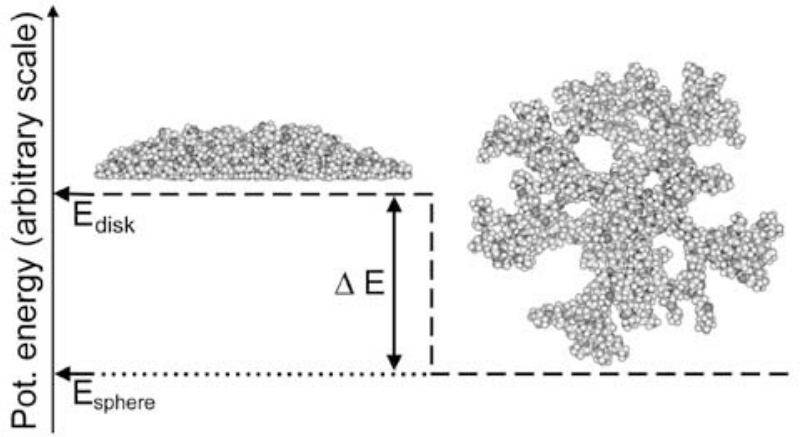

Fig. 10. Illustration of the relation between the potential energies of a spherical dendrimer, $E_{\text {sphere, }}$, and a compressed dendrimer, $E_{\text {disk }}$. The deformation energy is the difference $E_{\text {disk }}-E_{\text {sphere }}=\Delta E$. Energy fluctuations are small on this scale (between 1 and $5 \%$ of $\Delta E$ ).

the $q=-0.5 e$ configuration. This magnitude of $q$ is the smallest one that leads to completely flattened configurations for G2-G5 PAMAM models. A comparison between this configuration and an AFM profile of an adsorbed dendrimer is show in Figure 8 for generation 5 . The agreement is reasonable and we will continue to use this strength of $E_{\text {confine }}$ to provide the dendrimer deformation in the discussion that follows.

Figure 9 shows typical images of the two types of PAMAMs after flattening. Due to the nature of the confining potential the positively charged amine-terminated PAMAMs show a much higher degree of deformation. In this case different runs of the same simulation follow closely similar paths, and the resulting flattened dendrimer conformations are very reproducible.

On the other hand, the acetamide variety deforms much less. In the absence of charged end groups there are numerous different irregular compact dendrimer conformations each representing one local minimum in the potential energy surface. As a consequence, the observed conformations for a particular run depend on the initial location and orientation of the molecule. Through repeated annealing transitions between different conformations can be explored, however the shapes shown in Figure 9 as well as the potential energies remain very similar within the range of thermal fluctuations.

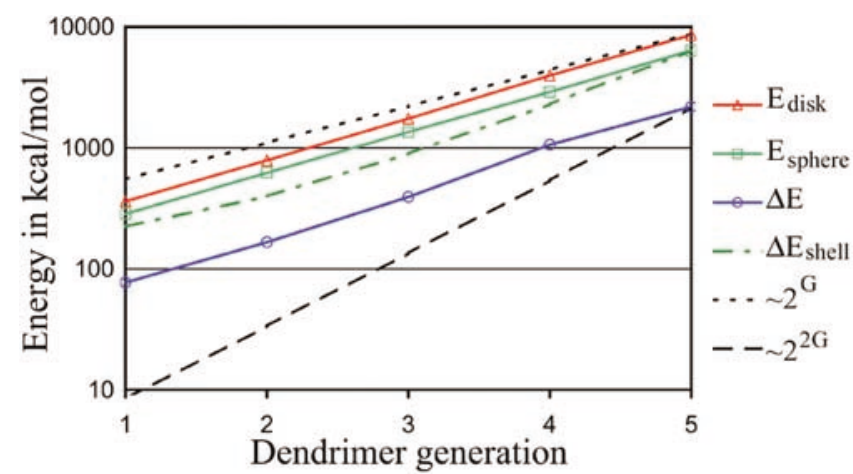

Fig. 11. Potential energies for spherical amine-PAMAMs, flattened amine-PAMAMs $(q=-0.5)$ and energy difference, $\Delta E$, between the two conformations (see also Fig. 8). Error bars are comparable to the symbol size.

To rule out the influence of edge effects due to the finite size of the model surface, a G3-amine PAMAM was simulated both on the full-size grid and on one with a surface area reduced by a factor of four. No significant difference was found in shape or conformational energy of the dendrimer.

Comparing the conformational energies between the spherically symmetric dendrimers and the compressed ones provides a measure of the stress in the molecules in their flattened state, see Figure 10. This energy of deformation, $\Delta E$, is evaluated for $q=-0.5$ and depends on the dendrimer generation. (Note that the confining potential, i.e. the second term of Eq. (1), is not expected to correspond to a physically meaningful quantity and therefore has to be excluded.) The average values of $\Delta E$ for the amine dendrimers shown in Figure 9b are plotted in Figure 11 as a function of generation G. The observed scaling of $\Delta E$ with $\mathrm{G}$ only occurs for flattened structures. Due to the flexibility of these hyper-branched molecules, the deformation energy $\Delta E$ remains low at minor deformations (Fig. 9a). In these cases, although the value of the confining potential ( $E_{\text {confine }}$ in Eq. (1), not shown in Fig. 11) is still significant, the induced stress, $\Delta E$, in the molecule is relatively small and comparable to the thermal fluctuations during the simulation (data not shown).

It is expected that dendrimers of higher generation are less deformable making it more and more difficult for 


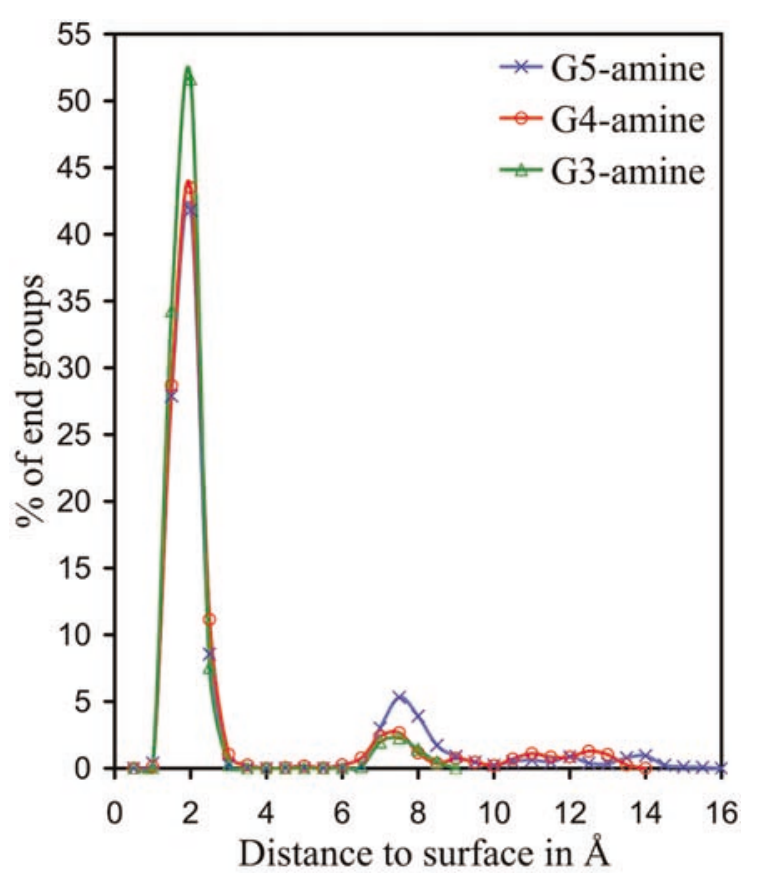

Fig. 12. Distributions of end groups as a function of height above substrate for maximally deformed $(q=-0.5)$ generation 3-5 amine PAMAM dendrimers.

branch ends to make contact with the surface. This was examined by the Monte Carlo studies of Mansfield [20] as well as experimentally verified using AFM [17]; however, we have been unable to reliably explore the higher generations with atomistic molecular dynamics because of computational demands. For the lower-generation dendrimers studied here this increase in rigidity is not observed. A means by which quantification of the deformation can be performed is to examine the distribution of the distances between end groups and the surface plane. In Figure 12 the distance distributions of end groups to the surface plane for flattened $(q=-0.5)$ generation 3-5 amine PAMAMs are compared. The fraction of branch ends that are blocked from touching the surface increases from $6 \%$ to $21 \%$ from generation 3 to 5 , but most of the end groups can still be found in direct contact with the substrate.

The behavior of the acetamide-terminated dendrimers is qualitatively similar to that of the amine-terminated PAMAMs. The deformation is not as dramatic as in the amine case, see Figures 9, 13 and 14. At high magnitude of $q$, however, many branch ends are also in direct contact with the surface.

\section{Discussion}

In an isotropic medium, dendrimers are considered to be spherical objects. There has, however, been much discussion in the literature about the exact density distribution within the molecule. Some authors use a "dense shell" description of dendrimers with most of the end groups on the dendrimer surface [29]. Others favor the "dense core" approach, which predicts backfolding of branches

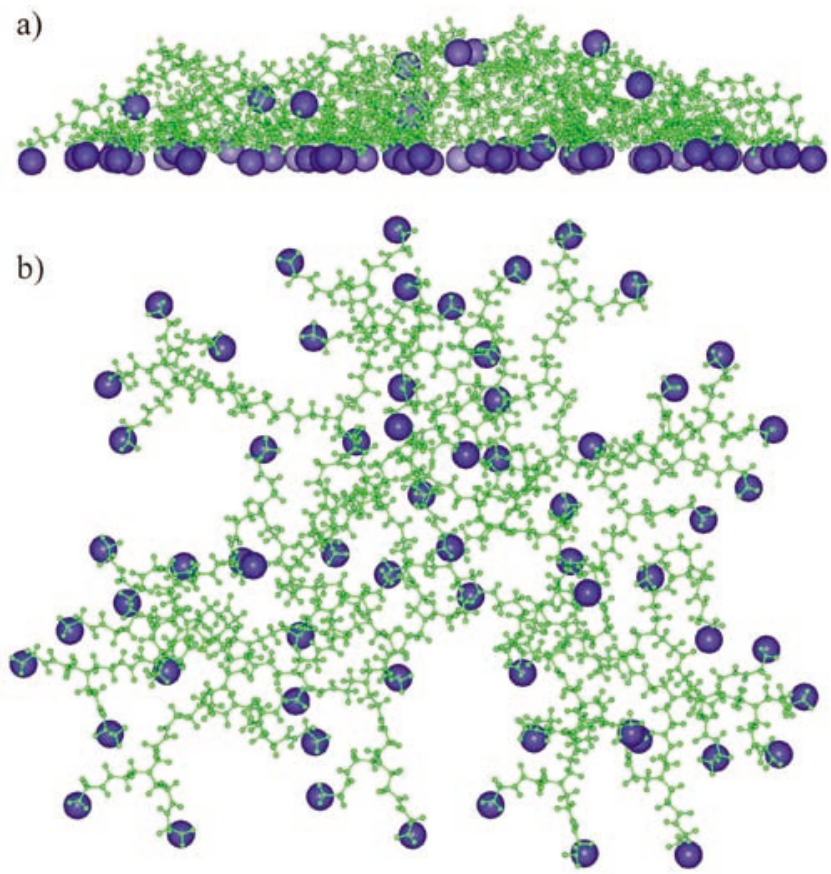

Fig. 13. Side view (a) and top view (b) of a flattened G4amine PAMAM $(q=-0.5)$. Nitrogen atoms located at the branch ends are shown as spheres.

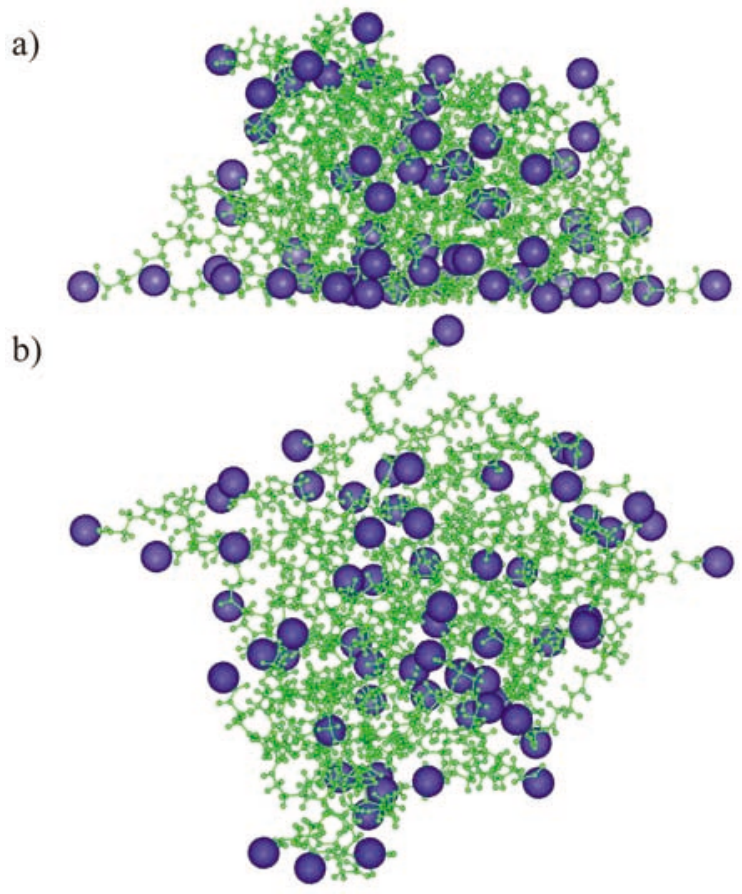

Fig. 14. Side view (a) and top view (b) of a flattened G4acetamide PAMAM $(q=-0.5)$. Carbon atoms located at the branch ends are shown as spheres. 
resulting in a higher density at the core [30-37]. Many detailed studies on specific dendrimers show that interactions between functional groups on the dendrimer as well as the solvent quality strongly influence the resulting shape of the molecule, $[21,28,38-46]$ while in a small-angle neutron scattering experiment Nisato et al. did not find any changes in dendrimer size as a function of $p \mathrm{H}$ or salt concentration [47]. In our simulations of free dendrimers the distribution of end groups does depend strongly on the charge state of the end group functionality (in agreement with experimental data for amine- and acetamideterminated G5 PAMAMs [28]) which will vary with the chemical species as well as the acidity of the environment.

The AFM analysis demonstrates that charged and uncharged dendrimers both strongly adsorb to mica. The presence of solvent does cause swelling to some degree, however, significant flattening still occurs. Comparison of the apparent size of PAMAM dendrimers imaged in water vs. in air, Figure 3 and Table 1, shows their difference in height, $h_{\text {water }}=2 h_{\text {air }}$, and volume, $V_{\text {water }}=2.5 V_{\text {air }}$. A potential concern regarding this type of volume analysis is the effect tip convolution may have. There are two reasons to believe that this effect alone does not explain the observed volume increase in water. First, there is a distinct change in height, which is independent of tip radius and consistent with reference [19]. Secondly, direct measurement of the tip radii during their respective scans indicated that they were about $5 \mathrm{~nm}$ in both air and water. After taking into account tip broadening [17,18] a lower limit for the volume increase is $V_{\text {water }} \geqslant 1.6 V_{\text {air }}$.

It is instructive to consider how many water molecules per amine and ketone group are present. A G7-dendrimer has 3062 nitrogen and oxygen atoms. Using a density of $1.1 \mathrm{~g} / \mathrm{cm}^{3}$, the observed volume increase implies, on average, 1-3 $\mathrm{H}_{2} \mathrm{O}$ per functional group, thus shielding surrounding atoms from their partial electric charges.

One difficulty with trying to make a connection between AFM experiments and simulations is the size of the molecule in question: AFM gives more accurate measurements for higher-generation dendrimers (G8, G9), but simulation times increase exponentially with generation making it harder and harder to obtain enough data for these large models. For G5 PAMAMs, comparison of experimental AFM data with simulation results (see Fig. 8) shows qualitative agreement between the resulting dendrimer profiles. This demonstrates that the molecular backbone of the dendrimers does allow deformation into very flat disks as seen in experiment.

The measured heights of acetamide dendrimers are only slightly greater than in the amine case. No significant difference in adsorption behavior on mica is observed. A thorough investigation into the influence of end group modification on biologic activity (Ref. [8]) will require more accurate methods than the ones used here.

The amount of energy required for the experimentally observed flattening and its dependence on generation $\mathrm{G}$ was examined in Figure 11. The question is, up to what generation $\mathrm{G}$ the exponential trend is expected to continue. The number of end groups $N$ and therefore the total
Table 2. Coefficients for conformational energies in Figure 10, assuming $E=E_{0} 2^{\gamma \mathrm{G}}$.

\begin{tabular}{cccc}
\hline & $E_{\text {disk }}$ & $E_{\text {sphere }}$ & $\Delta E$ \\
\hline$E_{0}(\mathrm{kcal} / \mathrm{mol})$ & 162 & 130 & 32 \\
\hline$\gamma$ & 1.15 & 1.12 & 1.23 \\
\hline
\end{tabular}

charge $Q$ of the molecule scales as $2^{\mathrm{G}}$, while the surface area of dendrimers grows only quadratically in G. From this it follows that after a certain generation (the so-called de Gennes limit), perfect dendrimer growth is no longer possible due to spatial constraints, i.e. too many branches occupying too little space.

Near the de Gennes limit, one would expect to see a change in dendrimer structure, stiffness and ability to deform. For the range of generations explored here, however, no such transition is observed, i.e. in Figure 11 all three calculated potential energies follow the same exponential trend from generations $2-5$. Assuming the conformational energies are of the form $E=E_{0} 2^{\gamma \mathrm{G}}$, the parameters $E_{0}$ and $\gamma$ listed in Table 2 are determined. Note that the energies grow faster than the number of end groups, $N=4 \cdot 2^{\mathrm{G}}$.

Since a large part of the potential energy is due to electrostatic interactions it is compared to the electrostatic energy $E_{\text {shell }}$ of a uniformly charged spherical shell with the same radius $r_{\text {dendrimer }}$ and total charge $Q$ as the dendrimer. In the simulations a dielectric constant was used to mimic the screening of charges by the solvent. Therefore, $E_{\text {shell }}$ is proportional to $Q^{2} / r_{\text {dendrimer }}^{2}$ (as opposed to $Q^{2} / r_{\text {dendrimer }}$ in the absence of screening) or approximately

$$
E_{\text {shell }} \sim \frac{2^{2 \mathrm{G}}}{\left(r_{\text {core }}+\mathrm{G} r_{\text {monomer }}\right)^{2}}
$$

In Figure 11 this function is shown as a dot-dashed line. For small generations $E_{\text {shell }}$ follows the trend seen in the simulation data much more closely compared to the lines of the form $2^{\mathrm{G}}$ and $2^{2 \mathrm{G}}$ or the potential energy of a charged shell without screening.

Using simple geometric considerations an argument can be made as to why no deviation in the dependence of the energy on $\mathrm{G}$ for generations $<6$ is expected. In order to add a new generation, $\mathrm{G}+1$, of monomers one needs to attach $N_{\mathrm{G}+1}=4 \cdot 2^{\mathrm{G}+1}$ branches. The maximum possible surface area to accommodate this many new branches on a dendrimer at generation $\mathrm{G}$ is $4 \cdot \pi\left(r_{\text {core }}+\mathrm{G} r_{\text {monomer }}\right)^{2}$, where $r_{\text {core }}$ is the radius of the dendrimer core and $r_{\text {monomer }}$ the effective additional radius of each generation of monomers. Let $M$ be the maximum number of carbon chains that can be attached to the outside of the existing dendrimer. One can calculate $M$ by determining the number of hard spheres of radius $r_{\text {carbon }}$ that can be packed onto the dendrimer surface of radius $r_{\text {dendrimer }} \gg r_{\text {carbon }}$. 
$M$ is approximately given by

$$
\begin{aligned}
M= & \frac{2 \pi}{\sqrt{3}}\left(\frac{r_{\text {dendrimer }}}{r_{\text {carbon }}}+1\right)^{2}= \\
& \frac{2 \pi}{\sqrt{3}}\left(\frac{r_{\text {core }}+\mathrm{G} r_{\text {monomer }}}{r_{\text {carbon }}}+1\right)^{2} .
\end{aligned}
$$

Once $N_{\mathrm{G}+1}$ is larger than $M$, the upper limit for perfect dendrimer growth has been reached. Assuming $r_{\text {carbon }}=$ $0.17 \mathrm{~nm}, r_{\text {core }}=0.9 \mathrm{~nm}, r_{\text {monomer }}=0.9 \mathrm{~nm}, N_{\mathrm{G}+1}$ does not exceed $M$ up until generation 10. Even though this argument ignores branch folding and imperfections during earlier stages of dendrimer growth, it makes a good rule of thumb for dense shell models of low-generation dendrimers.

Presumably, for actual polymers adsorbed to a substrate a significant amount of the surface binding energy will be provided by hydrogen bonds. It is interesting to estimate the energy that can be gained by such interactions and compare it to the numerical result for the energy lost to elastic deformation, $\Delta E$. The binding energy for typical H-bonds is $3-10 \mathrm{kcal} / \mathrm{mol}$. Assuming that such bonds are primarily formed between the end groups and the surface, this would result in a contribution to the total binding energy of $192-640 \mathrm{kcal} / \mathrm{mol}$ for a G4 PAMAM (which has 64 end groups). Comparing this to the numerically determined $\Delta E$ of $1000 \mathrm{kcal} / \mathrm{mol}$, one can see that $\mathrm{H}$ bonds could contribute $20-60 \%$ of the surface interaction energy. This indicates that the experimental deformation is both mechanically achievable and energetically possible through a combination of electrostatic interaction and H-bonding with the mica surface.

\section{Conclusions}

Both charged and uncharged PAMAM dendrimers strongly adsorb to mica surfaces resulting in deformation of the molecules. The heights of the molecules as determined by AFM are much less than the diameter in their spherically symmetric state and do not depend strongly on the charge of the branch ends. Flattening of dendrimers also occurs at the liquid-solid interface, although to a lesser degree. In aqueous environments swelling of the dendrimers results in an increase in measured heights and volumes.

Molecular dynamics simulations are used to investigate the structure of dendrimers in their spherically symmetric as well as compressed state. The model dendrimers investigated here are shown to be highly flexible and capable of forming very flat structures with little free volume left inside the polymer. This deformation allows for multiple interaction sites between branch ends and the substrate. These results are consistent with experimental observations using AFM. The energy of deformation increases exponentially with generation G. No deviation from this scaling law was found for generations $2-5$, i.e. no increase in stiffness is observed for these small generations. A simple model for determining the de Gennes limit indicates that this is to be expected.
This project has been funded with federal funds from the National Cancer Institute, National Institutes of Health, under Contract \# NOI-CO-97111. The computations were performed in part on the National Science Foundation Terascale Computing System at the Pittsburgh Supercomputing Center. We appreciate the helpful discussions and the assistance of Troy Wymore at PSC with some of the simulations.

\section{References}

1. A.W. Bosman, H.M. Janssen, E.W. Meijer, Chem. Rev. 99, 1665 (1999).

2. J. Haensler, F.C. Szoka jr., Bioconjugate Chem. 4, 372 (1993).

3. A. Bielinska, J.F. Kukowska-Latallo, J. Johnson, D.A. Tomalia, J. Baker jr., Nucleic Acids Res. 24, 2176 (1996).

4. J.F. Kukowska-Latallo, A.U. Bielinska, J. Johnson, R. Spindler, D.A. Tomalia, J.R. Baker jr., Proc. Natl. Acad. Sci, USA 93, 4897 (1996).

5. J.P. Thompson, C.-L. Schengrund, Glycoconjugate J. 14, 837 (1997).

6. N. Malik, E.G. Evagorou, R. Duncan, Anti-Cancer Drugs 10, 767 (1999).

7. H. Maruyama-Tabata, Y. Harada, T. Matsumura, E. Satoh, F. Cui, M. Iwai, M. Kita, S. Hibi, J. Imanishi, T. Sawada, O. Mazda, Gene Therapy 7, 53 (2000).

8. A. Quintana, E. Raczka, L. Piehler, I. Lee, A. Myc, I. Majoros, K. Patri, T. Thomas, J. Mule, J.R. Baker jr., Pharm. Res. 19, 1310 (2002).

9. M. Wells, R.M. Crooks, J. Am. Chem. Soc. 118, 3988 (1996).

10. K. Takada, D.J. Díaz, H.D. Abruña, I. Cuadrado, C. Casado, B. Alonso, M. Morán, J. Losada, J. Am. Chem. Soc. 119, 10763 (1997).

11. V.V. Tsukruk, F. Rinderspacher, V.N. Bliznyuk, Langmuir 13, 2171 (1997).

12. V.V. Tsukruk, Adv. Mater. 10, 253 (1998).

13. V.N. Bliznyuk, F. Rinderspacher, V.V. Tsukruk, Polymer 39, 5249 (1998).

14. A. Hierlemann, J.K. Campbell, L.A. Baker, R.M. Crooks, A.J. Ricco, J. Am. Chem. Soc. 120, 5323 (1998).

15. H. Tokuhisa, M. Zhao, L.A. Baker, V.T. Phan, D.L. Dermody, M.E. Garcia, R.F. Peez, R.M. Crooks, T.M. Mayer, J. Am. Chem. Soc. 120, 4492 (1998).

16. J. Li, L.T. Piehler, D. Qin, J.R. Baker, D.A. Tomalia, Langmuir 16, 5613 (2000).

17. T.A. Betley, M.M. Banaszak Holl, B.G. Orr, D.R. Swanson, D.A. Tomalia, J.R. Baker jr., Langmuir 17, 2768 (2001).

18. T.A. Betley, J.A. Hessler, A. Mecke, M.M. Banaszak Holl, B.G. Orr, S. Uppuluri, D.A. Tomalia, J.R. Baker jr., Langmuir 18, 3127 (2002).

19. T. Müller,, D.G. Yablon, R. Karchner, D. Knapp, M.H. Kleinmann, H. Fang, C.J. Durning, D.A. Tomalia, N.J. Turro, G.W. Flynn, Langmuir 18, 7452 (2002).

20. M.L. Mansfield, Polymer 37, 3835 (1996).

21. I. Lee, B.D. Athey, A.W. Wetzel, W. Meixner, J.R. Baker jr., Macromolecules 35, 4510 (2002).

22. M.F. Ottaviani, F. Montalti, M. Romanelli, N.J. Turro, D.A. Tomalia, J. Phys. Chem. 100, 11033 (1996).

23. R.C. van Duijvenbode, M. Borkovec, G.J.M. Koper, Polymer 39, 2657 (1998). 
24. S. Lifson, A.T. Hagler, P. Dauber, J. Am. Chem. Soc. 101, 5111 (1979).

25. B.R. Brooks, R.E. Bruccoleri, B.D. Olafson, D.J. States, S. Swaminathan, M. Karplus, J. Comp. Chem. 4, 187 (1983).

26. A.D. MacKerell jr., B. Brooks, C.L. Brooks III, L. Nilsson, B. Roux, Y. Won, M. Karplus, CHARMM: The Energy Function and Its Parameterization with an Overview of the Program, The Encyclopedia of Computational Chemistry, edited by P.v.R. Schleyer et al. Vol. 1 (John Wiley \& Sons, Chichester, 1998) pp. 271-277.

27. C.L. Jackson, H.D. Chanzy, F.P. Booy, B.J. Drake, D.A. Tomalia, B.J. Bauer, E.J. Amis, Macromolecules 31, 6259 (1998).

28. I.J. Majoros, B. Keszler, S. Woehler, T. Bull, J.R. Baker jr., Macromolecules 36, 5526 (2003).

29. P.G. de Gennes, H. Hervet, J. Phys. Lett. 44, L351 (1983).

30. R.L. Lescanec, M. Muthukumar, Macromolecules 23, 2280 (1990).

31. M.L. Mansfield, L.I. Klushin, J. Phys. Chem. 96, 3994 (1992).

32. M.L. Mansfield, K.I. Klushin, Macromolecules 26, 4262 (1993).

33. M. Murat, G.S. Grest, Macromolecules 29, 1278 (1996).

34. D. Boris, M. Rubinstein, Macromolecules 29, 7251 (1996).

35. Z.Y. Chen, S.-M. Cui, Macromolecules 29, 7943 (1996).
36. T.J. Prosa, B.J. Bauer, E.J. Amis, D.A. Tomalia, R. Scherrenberg, J. Polym. Sci. B 35, 2913 (1997).

37. M.L. Mansfield, Macromolecules 33, 8043 (2000).

38. G.R. Newkome, J.K. Young, G.R. Baker, R.L. Potter, L. Audoly, D. Cooper, C.D. Weis, Macromolecules 26, 2394 (1993).

39. S. Uppuluri, S.E. Keinath, D.A. Tomalia, P.R. Dvornic, Macromolecules 31, 4498 (1998).

40. A.M. Naylor, W.A. Goddard, G.E. Kiefer, D.A. Tomalia, J. Am. Chem. Soc. 111, 2339 (1989).

41. D.A. Tomalia, A.M. Naylor, W.A. Goddard, Angew. Chem. Int. Ed. Engl. 29, 138 (1990).

42. P. Miklis, T. Çagin and W.A. Goddard III, J. Am. Chem. Soc. 119, 7458 (1997).

43. L. Cavallo, F. Fraternali, Chem. Eur. J. 4, 927 (1998).

44. R. Scherrenberg, B. Coussens, P. van Vliet, G. Edouard, J. Brackman, E. de Brabander, K. Mortensen, Macromolecules 31, 456 (1998).

45. P. Welch, M. Muthukumar, Macromolecules 31, 5892 (1998).

46. A. Topp, B.J. Bauer, D.A. Tomalia, E.J. Amis, Macromolecules 32, 7232 (1999).

47. G. Nisato, R. Ivkov, E.J. Amis, Macromolecules 33, 4172 (2000). 